\title{
OGO SATELLITE WAKE STRUCTURE DEDUCED FROM ANTENNA IMPEDANCE MEASUREMENTS
}

\author{
H. WEIL and R. G. YORKS \\ Radioastronomy Observatory, University of Michigan, Ann Arbor, Mich. 48104, U.S.A.
}

(Received 16 December 1969)

\begin{abstract}
Measurements of the complex impedance of an 18-m monopole antenna at $2.5 \mathrm{MHz}$ are used to study the electron densities in and out of the wake of a large spinning satellite in the Earth's upper ionosphere. Some spacecraft orientation information is also obtained from the impedance data.

The ratios of the wake electron densities, averaged over the length of the antenna, to the unperturbed local densities are calculated for all angles of attack of the satellite. Electron density distributions along the antenna are derived.
\end{abstract}

\section{INTRODUCTION}

When ionospheric sounding devices are carried aboard satellites or high altitude rockets, it is necessary to take into account the fact that the vehicle will perturb the ionosphere. A highly nonuniform rarefied wake with a negatively charged core is formed behind the vehicle which is different in structure from the thin electron deficient sheath which forms about stationary objects in a plasma. In addition to the considerable intrinsic interest in this phenomenon and its practical effect on in situ ionospheric measurements, this wake is a source of enhanced radar return from space vehicles. Although an extensive body of theoretical work on the wake structure has developed in the literature, the amount of quantitative work, both calculated and experimental, is limited. Surveys of the field are presented by Samir and Willmore (1965), Al'Pert (1965) and Liu (1969).

The present paper discusses an experimental probing of the wake behind the Orbiting Geophysical Observatory II (OGO-II) illustrated in Fig. 1 as a by-product of a radio astronomy experiment. This wake probing aspect of the experiment was not planned, in fact it was made possible only by a failure of the spacecraft stabilization system to maintain the planned three axis stabilization. The ionospheric probe was an $18 \mathrm{~m}$ monopole antenna. A $2.5 \mathrm{MHz}$ impedance bridge measured the antenna impedance on a time sharing basis taking samples every $0.288 \mathrm{sec}$ at the slowest data rate. The spacecraft motion rotated the antenna cyclically from a position behind the vehicle pointing downstream, to pointing upstream, and back to downstream in a period of approximately four minutes, or roughly ${ }_{26}^{1}$ of an orbit period. Since antenna impedance is a sensitive indicator of the ambient electron density we were thus provided with a probe of the wake electron density compared to that in a relatively unperturbed ionosphere.

In addition to the results described in this paper there are recent results on wake structure behind a sounding rocket by Stone, Fainberg and Alexander (1969) where antenna impedance measurements were also used as the probing tool.

For a qualitative picture of the relative dimensions of the antenna and wake we use theoretical-numerical results on the electron density distributions in the wake of an arbitrarily oriented prolate spheroid (Sawchuck, 1963). These results are for the altitude, body speed and size of the order of those for OGO-II where we observe wake effects. These are the only numerical results for non-symmetrical bodies at different angles of attack and are 
therefore useful for the present comparison even though they are not accurate near the body since they are not based on a self consistent theory (the ion distribution used in Poisson's equation for the potential is an approximate distribution for neutrals). A self consistent analysis would yield a greater depletion of electron density in the very near wake (Liu, 1969).

Sawchuck presented figures showing constant electron density contours for a prolate spheroid with axes $4 \mathrm{~m}$ and $2 \mathrm{~m}$ traveling at a $500 \mathrm{~km}$ altitude at $8 \mathrm{~km} / \mathrm{sec}$. They correspond to a satellite speed approximately seven times the r.m.s. thermal speed of the ambient ions. Figures 2(a-c) are from Sawchuck's paper and they show his computed curves of constant electron density, $n_{e}$ for a prolate spheriod. We have taken the spheroid to represent the OGO-II body and solar paddles, and in addition show the attached cylindrical antenna. The three figures represent the different angles of attack, $\alpha=0^{\circ}, 45^{\circ}$ and $90^{\circ}$. In the first case, that of zero angle of attack, the antenna itself does not greatly affect the wake structure, while for the other two orientations one would expect a small wake generated by the antenna. Since the antenna radius is very small compared to the spheroid dimensions we have neglected its contribution to the wake as well as contributions by other booms on the OGO.

In Fig. 2(c), the antenna end is out of the perturbed region for this broadside orientation, while in the trailing position shown in Fig. 2(a) the end is immersed in the wake.

Before discussing the detailed procedure for extracting wake information from the data, the intended primary radio astronomy experiment and the instrumentation will be described. This will indicate how the unexpected wake probing is directly of value for a subsequent similar radio astronomy experiment.

\section{PRIMARY EXPERIMENT AND INSTRUMENTATION}

The OGO-II satellite was launched on October 14, 1965 from the Western Test Range at Vandenberg Air Force Base, California, into an orbit with an inclination to the equator of $87^{\circ} \cdot 36$, with perigee and apogee altitudes of $413 \mathrm{~km}$ and $1512 \mathrm{~km}$ respectively, and a period of 104 minutes (TRW, 1965). The satellite was to be stabilized with one main body face always looking at the Earth, an orbiting plane experiment package looking in the direction of travel, and the solar paddles always directed towards the Sun. Horizon scanner problems caused rapid consumption of the stabilization gas supply and after 10 days of stabilized operation the spacecraft was spun-up to about $0.11 \mathrm{rev} / \mathrm{min}$ with the spin axis roughly perpendicular to the orbit plane. After several months the spin rate had increased and stabilized at about $0 \cdot 27 \mathrm{rev} / \mathrm{min}$. OGO-II carried 20 experiments for measurements of particles, fields, and auroras in the vicinity of the Earth.

The University of Michigan Radio Astronomy Observatory has an experiment on board to attempt to make a radio sky brightness map at $2.5 \mathrm{MHz}$. Figure 3 is a block diagram of the instrumentation showing the four main experiment channels: a $2.5 \mathrm{MHz}$ radiometer, a $2.0 \mathrm{MHz}$ radiometer, and a $2.5 \mathrm{MHz}$ impedance bridge which terminates in the blocks labeled 'sine phase detector' and 'cos phase detector'. The antenna for the system shown in Fig. 1 extended out from the end of the lower left solar paddle, is an 18-m long, $1.42 \mathrm{~cm}$ dia. monopole.

Since the entire orbit of OGO-II was spent in the Earth's upper ionosphere, it was planned to make use of the focusing properties of the ionosphere to effectively sharpen the antenna beam (Walsh and Weil, 1968), since the beam width of the monopole (or any other electrically short antenna) is far too broad for mapping purposes. The antenna was used continuously and simultaneously with the radiometers and the impedance bridge.

The impedance measurements were needed to enable absolute values of sky brightness to 


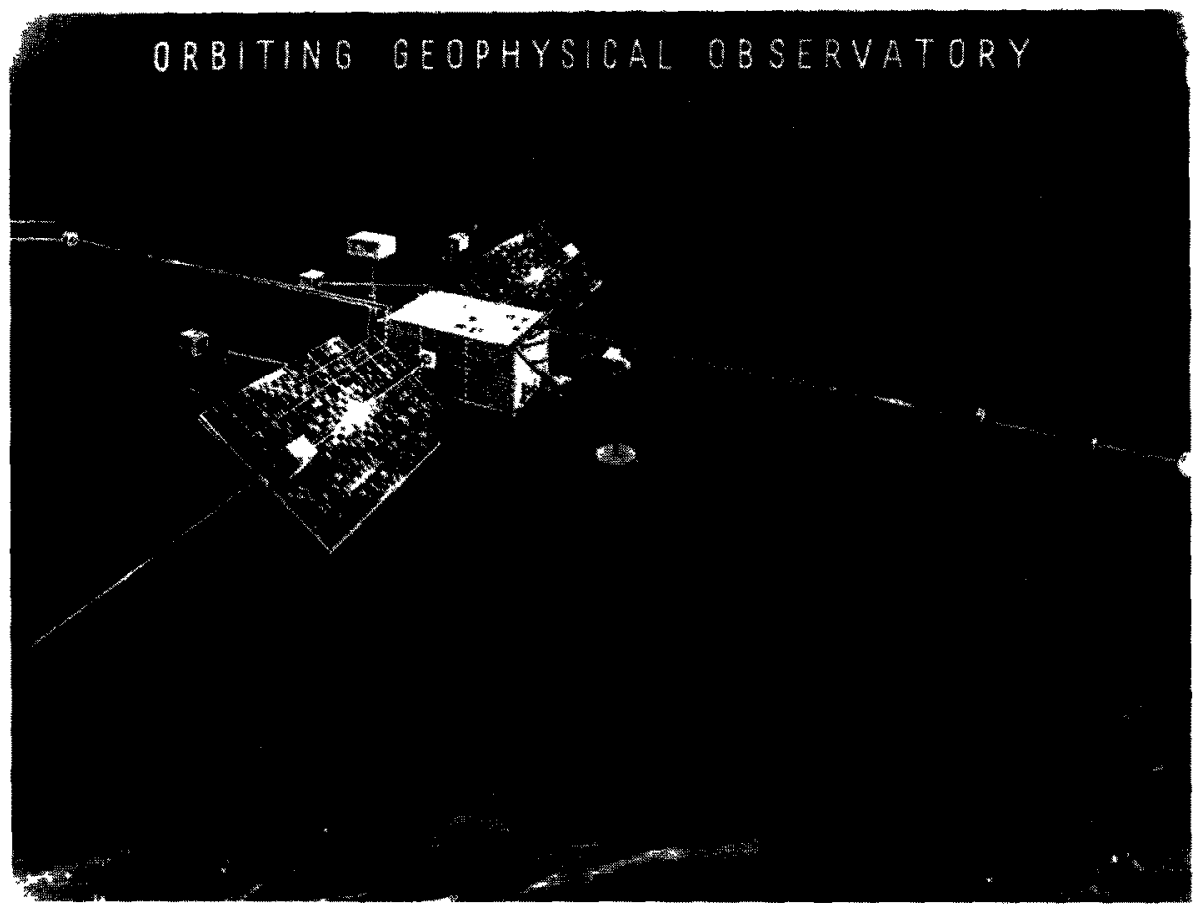

Fig. 1. The OGO-II satellite. 


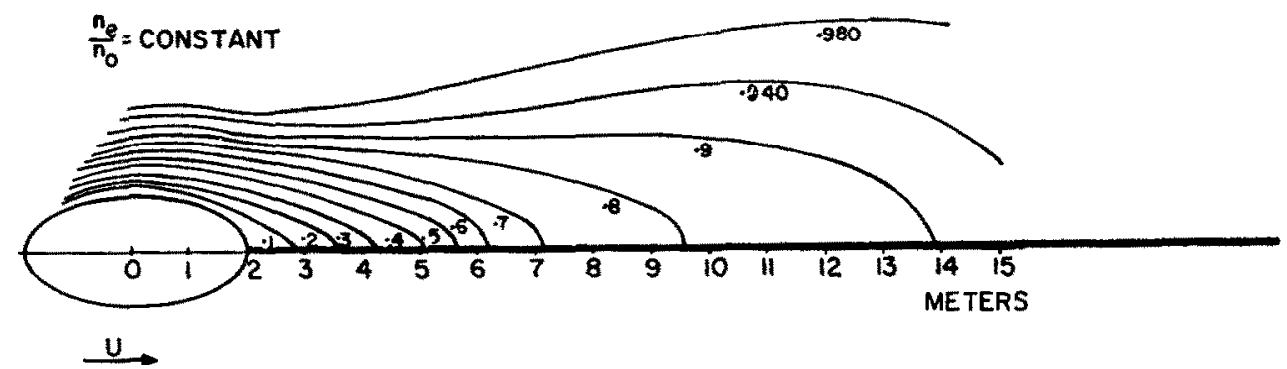

ANGLE OF ATTACK $=0^{\circ}$

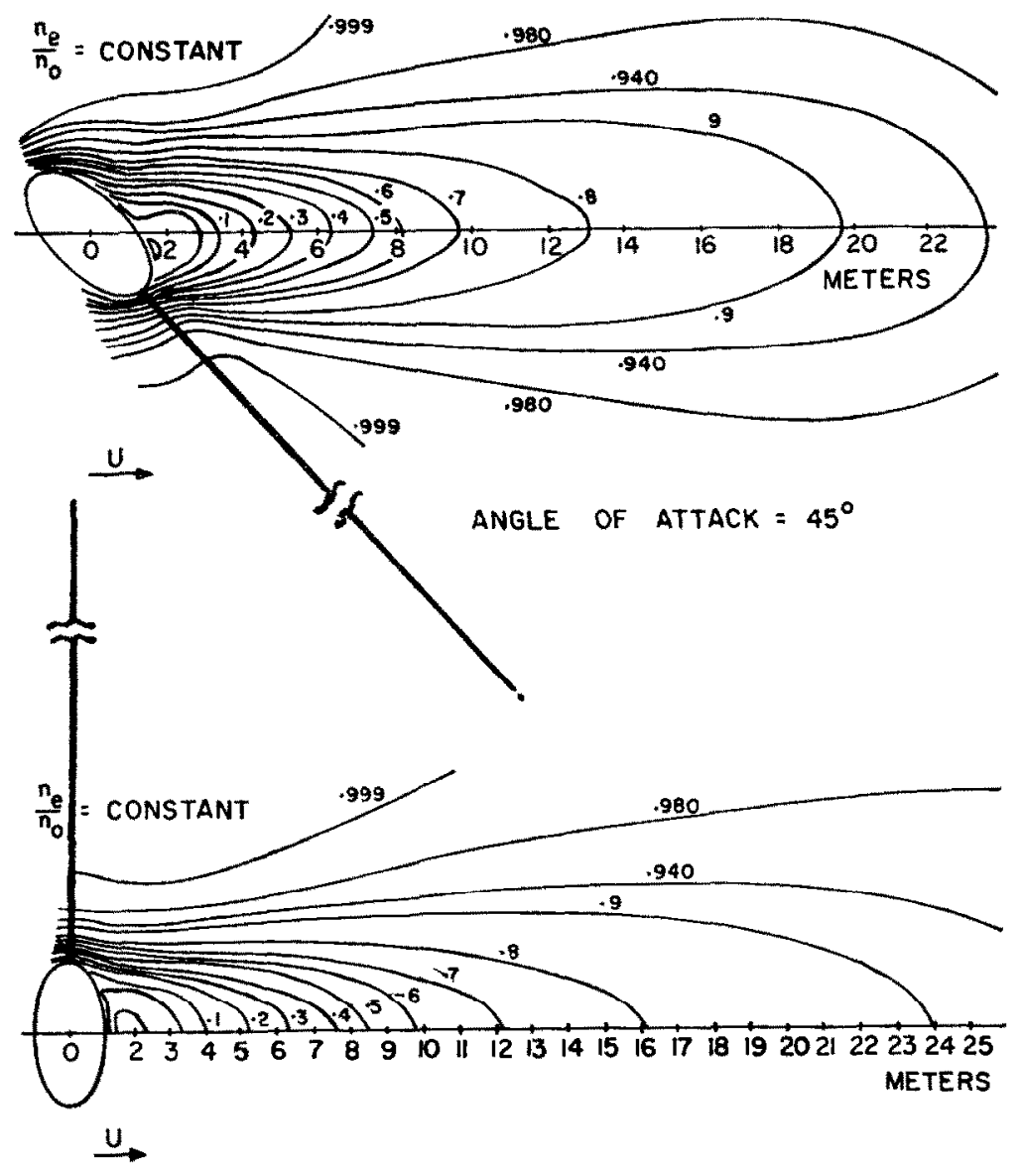

$$
\text { ANGLE OF ATTACK }=90^{\circ}
$$

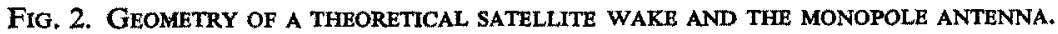

The model wake, illustrated by contours of constant electron density relative to the unpertur-

bed electron density, is from Sawchuck (1963). 


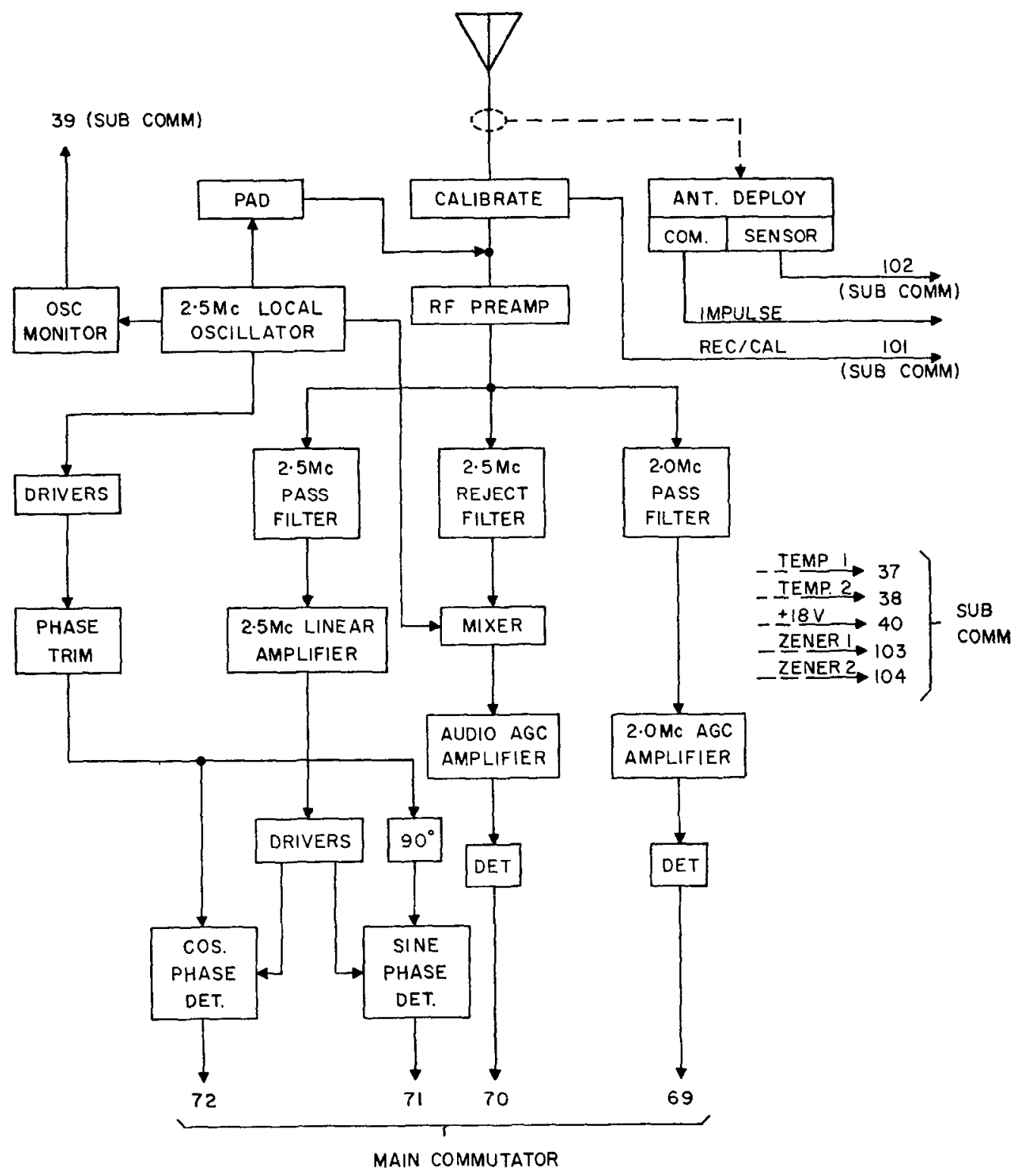

Fig. 3. BLOCK DiAgRam OF THE EXPERIMENT INSTRUMENTATION.

be calculated from the radiometer data. The principles involved in this type of sky brightness measurement are given, for example, by Walsh and Haddock (1965). The impedance bridge yields both resistive and reactive components of the antenna impedance and will give both positive and negative reactances. The range of impedance values measurable was from less than $100 \Omega$ to greater than $10,000 \Omega$. The $2.5 \mathrm{MHz}$ sine wave voltage level injected onto the antenna for the bridge measurement was less than $1 \mathrm{mV}$ r.m.s. The antenna was direct-coupled to spacecraft ground through an inductor.

The level of interference generated by the spacecraft at $2.5 \mathrm{MHz}$ was sufficiently high to obscure much of the expected brightness data. However, the impedance bridge, which is 
insensitive to such interference, yielded excellent data. Because of the spacecraft tumbling, the data is of special value in two ways. The first is to obtain rclative elcctron density information in the wake. Second the effect of the wake on impedance, even without interpretation in terms of electron density structure, can be used to increase the accuracy of sky brightness data derived on a duplicate experiment on OGO-IV. OGO-IV is successfully stabilized so that the monopole antenna is continually in the wake during long intervals. In addition the radio interference has been reduced.

\section{PROCEDURE}

Both the real and imaginary parts of the impedance are quite sensitive to the ambient plasma. The reactive part is the most useful because of the easily observed changes in sign which occur when the vehicle passes from a region where the local upper hybrid frequency, $f_{u}=\left(f_{p}^{2}+f_{g}^{2}\right)^{1 / 2}$, (square root of the sum of the plasma frequency squared and the gyro frequency squared) passes from being less than to greater than the operating frequency, which in this experiment is $2.5 \mathrm{MHz}$. The reactance is sensitive primarily to the plasma in a roughly cylindrical region encompassing the spacecraft body, solar paddle, and the antenna, in which the field lines are mainly confined.

If the ionospheric plasma were unperturbed and locally uniform we could correctly apply the approximate formula, equation (1), for the reactance of an electrically short cylindrical monopole in uniform magnetoplasma which was derived by Balmain (1964) using a quasistatic theory.

in which

$$
Z=\frac{-j a}{2 \pi \omega \varepsilon_{0} K^{\prime} F^{1 / 2}}\left(\ln \frac{L}{\rho}-1-\ln \frac{a+F^{1 / 2}}{2 F}\right)
$$

$F=\sin ^{2} \theta+a^{2} \cos ^{2} \theta$ and $\theta$ is the angle between the antenna axis and the Earth's magnetic field,

$\omega=2 \pi$ times the operating frequency,

$\varepsilon_{0}=$ free space permittivity $=8.8542 \times 10^{-12} \mathrm{~F} / \mathrm{m}$,

$a^{2}=K^{\prime} \mid K_{0}$,

$K^{\prime}=1-\frac{X U}{U^{2}-Y^{2}}$,

$K_{0}=1-\frac{X}{U}$,

$X=\frac{f_{p}^{2}}{f^{2}}, \quad Y=\frac{f_{g}}{f}, \quad U=1-\frac{j v}{2 \pi f}$,

$\nu=$ collision frequency,

$L=$ length of monopole in $\mathrm{m}$,

$\rho=$ radius of monopole in $\mathrm{m}$,

and

$Z=$ the antenna reactance in $\Omega$.

Given $Z, Y, \theta, U, f$, and the dimensions of the antenna, one can then obtain a value for $X$ by an iteration process. The ambient magnetic field amplitude is available from magnetometers carried on the spacecraft and the reactance is available from our impedance bridge measurements. 
Our antenna is in a non-uniform medium, but if we apply this procedure an effective electron density can then be computed from:

$$
\bar{n}=\frac{\varepsilon_{0} m \omega^{2} X}{e^{2}} \text { electrons } / \mathrm{m}^{3}
$$

where

$$
\begin{aligned}
m & =\text { the electron mass }=9.1091 \times 10^{-31} \mathrm{~kg}, \\
e & =\text { the electron charge }=1.6021 \times 10^{-19} \mathrm{C} .
\end{aligned}
$$

We shall assume that, as a function $l$, radial distance from the satellite body

$$
\frac{n_{e}}{n_{0}}=f(l)=1-\mathrm{e}^{-\beta l},
$$

where $\beta$ is a real constant. This satisfies the required physical conditions at $l=0$ and $l=\infty$ and, in fact, can provide a close fit to the numerical values given by Sawchuck. Using $f(l)$ as a weighting function in the averaging gives

$$
\frac{\bar{n}}{n_{0}}=\frac{1}{L} \int_{0}^{L} f(l) \mathrm{d} l=\frac{1}{\beta L}\left(\mathrm{e}^{-\beta L}-1\right),
$$

from which $\beta$ can be determined and used with (3) to construct constant $n_{e} / n_{0}$ curves.

A problem concerns the use of (1) for the present antenna system. The free space electrical length at $2.5 \mathrm{MHz}$ is 0.15 wavelengths, long enough to render questionable the applicability of a theory based upon the assumption that the antenna is electrically short. An added complication is that the dimensions of the spacecraft, which form a 'ground plane' against which the antenna is working, are much less than a wavelength at $2.5 \mathrm{MHz}$. However, since we are trying to obtain only relative densities around the spacecraft, we feel these limitations do not seriously limit our use of Equation (1); we are unaware of a more suitable theoretical result.

Also because of a limitation in present antenna theory we have used the $n_{e} / n_{0}$ distribution as a weighting function.

A second problem resulted from satellite tumbling because there was not complete data on the instanteous orientation of the spacecraft with respect to the magntic field. There was, however, information on the orientation of the spin axis of the spacecraft. With this, plus information extracted from the antenna impedance records, we have been able to determine the antenna magnetic field orientation. The method for doing this is outlined in the next section, and the cosines of the angles thus obtained are plotted on Fig. 5.

\section{ANTENNA-MAGNETIC FIELD ANGLE}

The problem of determining the time varying angle $\theta$ between the Earth's magnetic field and the antenna could be resolved in one of several ways using the measured antenna impedance data. The method used ignores the precession of the spacecraft spin axis so the results will be in error by 10-12 deg (the half angle of the precession cone at this epoch), and it will assume that the OGO-II orbit is circular. The orbit is actually an ellipse, but the ratio of the axes is only 1.003. It will be evident in the next section that this accuracy for the angle is adequate. 
The center of the cone of precession of the spin axis is perpendicular to the plane of the orbit (TRW, 1966). The spin axis of the spacecraft is the body $Z$-axis, which is therefore also perpendicular to the orbit plane. The body $X$ and $Y$ axes are rotating in the orbit plane and consequently so is the antenna ( $-X$ body axis). In one orbit around the Earth, the velocity vector of the spacecraft goes through one complete rotation in space in the plane of the orbit. Since the antenna is rotating in the same plane as the spacecraft velocity vector, the orientation of the antenna at a time $t_{0}+(3.67 / 104) \Delta t$, is the same as that of the velocity vector at a time $t_{0}+\Delta t$, where $t_{0}$ is a reference time, 104 is the orbital period in minutes, 3.67 is the spacecraft spin period in minutes, $\Delta t$ is the time in minutes measured from the reference time $t_{0}$. To determine $t_{0}$, we use the antenna impedance data of Fig. 4 . There is a well defined periodic maximum which we correlate with the time when the antenna and the velocity vector are aligned at $180^{\circ}$ with each other. This provides the reference time, $t_{0}$. The time varying orientation of the antenna with the magnetic field is therefore determined.

The magnetic field and the velocity vector are both given on the attitude orbit tape in a

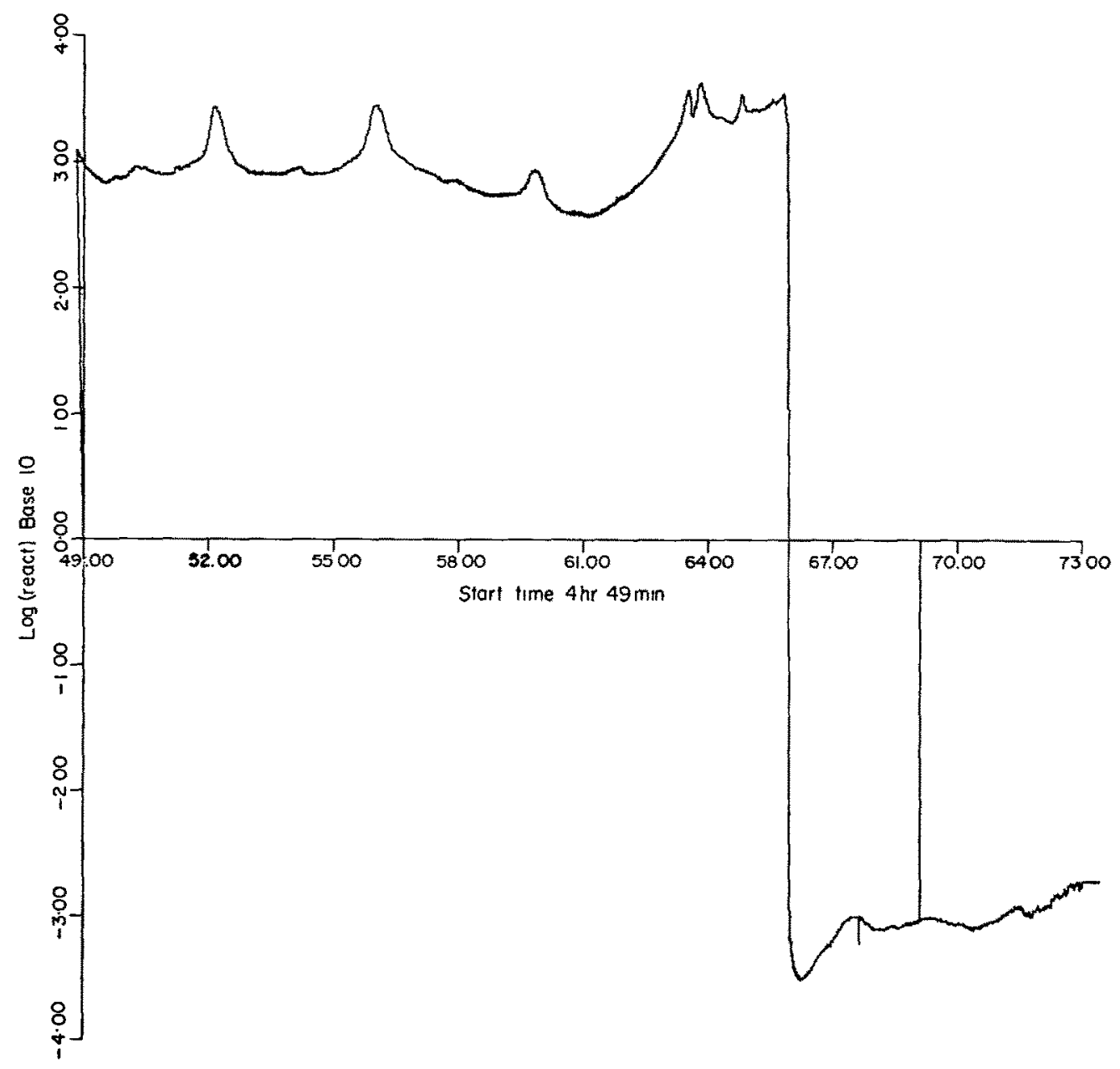

Fig. 4. Typical measured $\log _{10}$ (Reactance) vs, tome data. 
right hand system of coordinates, known as the geocentric equatorial inertial (GEI) coordinate system, where the $X$ - and $Y$-axes are in the Earth's equatorial plane, the $Z$-axis points northward, and the $X$-axis is directed towards the first point of Aries.

\section{RESULTS}

Figure 4 is a computer generated digital plot of the measured antenna reactance. In addition to the main reactance peaks, which are due to the antenna being in the wake, there are three secondary peaks in each spin cycle. Possible causes to consider for these secondary peaks are: (1) effects due to orientation of the antenna with the magnetic field, (2) regions both forward of the spacecraft and at right angles to the direction of motion, which are in some way depleted of electrons, (3) impedance changes in the antenna ground plane system as the booms and opposing solar paddles pass through the wake of the spacecraft.

We rule out explanation (1) because the antenna is neither lined up with, nor at right angles to, the magnetic field at times of the secondary peaks. In any case, since $Y^{2} \leq 0 \cdot 2$, magnetic field effects on the reactance are very small. We also discard (2) since it is very hard to conceive of a situation that would cause selective depletion of electrons only at $0^{\circ}$ and $90^{\circ}$. We are then left with case (3) as an explanation of the cause of these peaks. This is reasonable, since the spacecraft and its associated booms are an integral part of the antenna. If the wake effects are observable by the monopole they should also be observable, to some degree, by the 'ground plane', particularly by the opposing solar panel at $0^{\circ}$ and the booms at $\pm 90^{\circ}$.

On Fig. 5 we have plotted electron density corresponding to the reactance plotted in Fig. 4. We have, in addition plotted the cosine of the computed angle, $\theta$, between the antenna and the magnetic field direction. In order to check these computations of $\theta$, we have also plotted the cosine of the angle between the spacecraft velocity vector and the magnetic field direction, which is derived directly from data present on the attitude orbit tape. At the time of the greatest electron depletion, the angles of the antenna and of the velocity vector with the magnetic field direction should be the same. It can be seen in this figure that this is indeed the case.

Figure 5 is a digital plot of the electron density calculated for a portion of the time of Fig. 4, using Equations (1) and (2) above with

$$
\begin{gathered}
L=18.3 \mathrm{~m}, \quad \rho=0.0071 \mathrm{~m}, \\
f=2.5 \mathrm{MIIz}, \quad v-0 .
\end{gathered}
$$

Values of electron density have been calculated using various values of $v$ between 1000 and $10,000 \mathrm{rad} . / \mathrm{sec}$. The results on relative densities vary little from that with $\nu=0$, but the complexity of (1) increases significantly.

A model ionosphere electron density distribution was determined by assuming that the unperturbed electron density over this short time interval could be represented by a smooth curve fitted to maxima of the calculated wake density curve as shown in Fig. 5. Trial has shown that the principal features of Figs. 5-8, which are typical results of our data reduction, are not sensitive to the choice of smooth curve fit. In addition data samples too noisy for easy fitting were not used.

The ratio of the wake density to the unperturbed density, given by the fitted curves, is the effective relative electron density around the spacecraft, averaged over the length of the $18 \mathrm{~m}$ monopole. A plot of this averaged relative density is shown in Fig. 6(a). Zero degrees 




Fig. 5. UPPER CURVE: COSINE OF THE ANGLE BETWEEN THE ANTENNA AND THE MAGNETIC FIELD DIRECTIONS.

Lower curve: $\log _{10}$ (effective electron density) vs. time. These curves correspond to a portion of the data in Fig. 4.

corresponds to the direction ahead of the spacecraft and $180 \mathrm{deg}$ is trailing the spacecraft. Figure 6(b) shows calculated iso-electron density curves around the spacecraft, using the method outlined in the Procedure's section with the data from Fig. 6(a). We see that the electron depletion in this case reaches a maximum of 25 per cent. In some other cases the depletion reaches 30 per cent.

To interpret Figs. 6-8, it should be borne in mind that the relative densities associated with each distance along a radial line at a given value of the angle $\phi$ are the relative densities along the antenna when the angle of attack of the spacecraft, measured from the major axis, is $360^{\circ}-\phi$. The curvcs are therefore not equidensity contours throughout the wake corresponding to a fixed angle of attack. They would have been such curves if the vehicle had been symmetrical about the spin axis. 


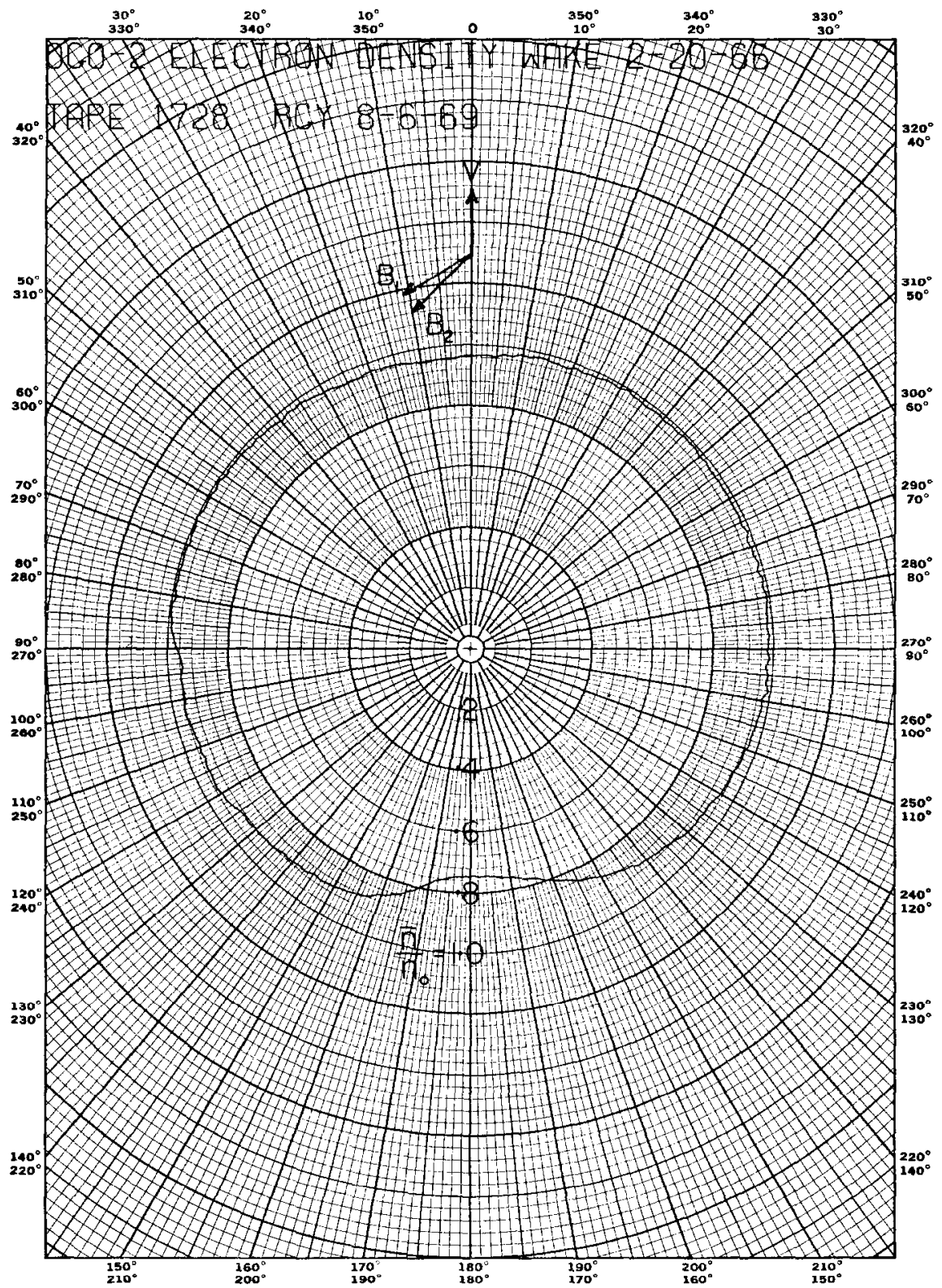

Fig. 6. (a) Polar Plot of efFeCtive electron Density Relative to the UNPERTURbed ELECTRON DENSITY FROM THE DATA OF FIG. 5.

Also shown is the direction $\mathbf{V}$ of the satellite velocity and the directions $\mathbf{B}_{1}$ and $\mathbf{B}_{\mathrm{a}}$ of the geomagnetic field at the start and finish of the spin cycle respectively. The directions of these quantities are also on all the subsequent figures. 




FiG, 6. (b) IsO-RELATIVE ELECTRON DENSITY CONTOURS FOR THE DATA OF 6(a).

The secondary peak ahead of the spacecraft is assumed to be a ground plane impedance effect due to the opposing solar paddle passing through the wake and not an electron depletion effect forward of the vehicle. The fluctuations in the contours are present because the data were used without smoothing and all telemetry and coding noise is present in the data records.

Figures 7 and 8 are further figures of the same type for other time intervals. They were chosen to illustrate possible magnetic field effects. On each figure the direction of the 


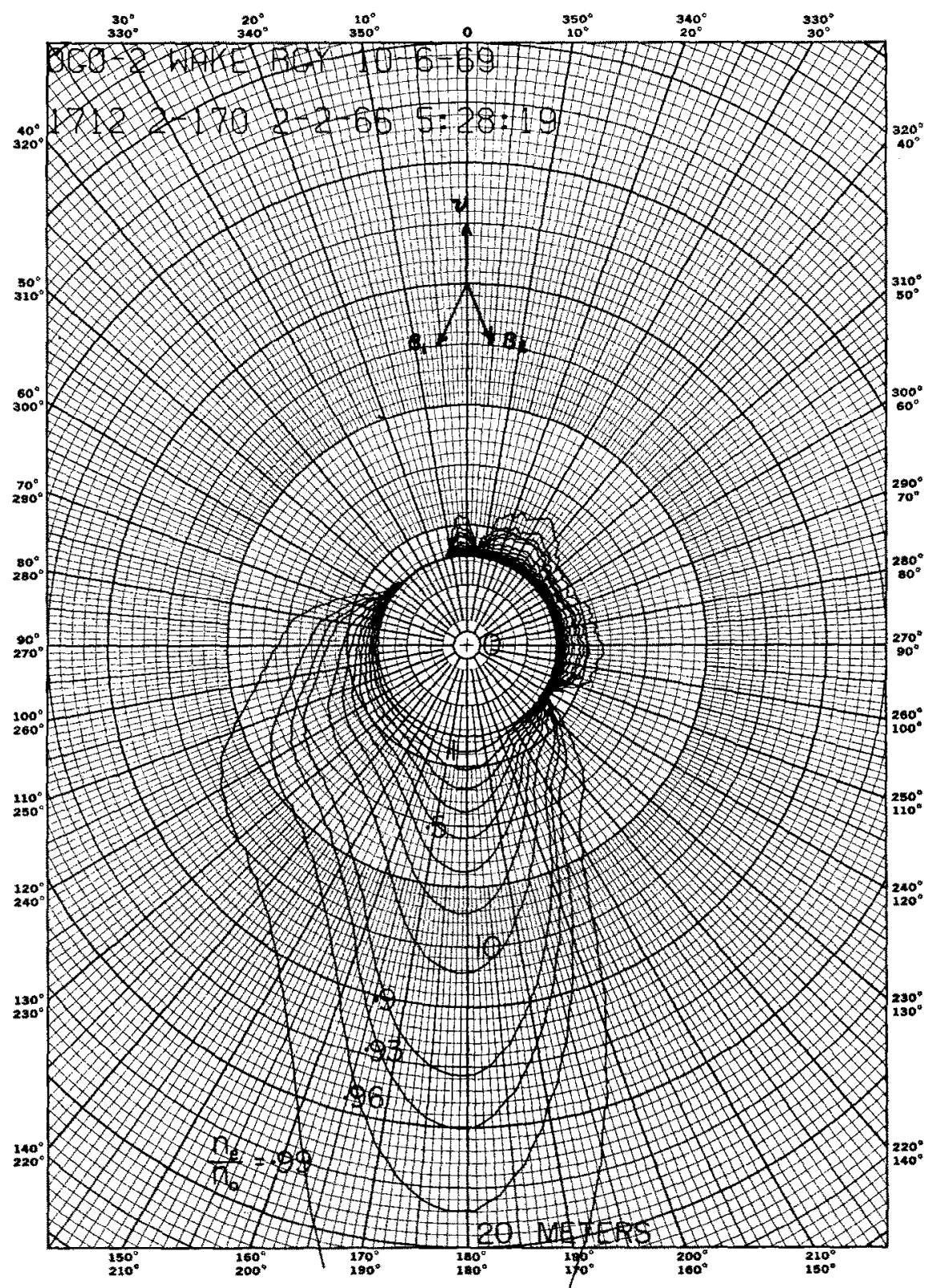

FIG. 7(a).

Fig. 7. Iso-Relative DeNsity CONTOURS ILlustrating two CASES WHEN $B_{1}$ AND $B_{2}$ ARE SYMMETRIC ABOUT $V$.

magnetic field at the beginning and end of the spin cycle is shown by arrows labeled $B_{1}$ and $B_{2}$ respectively. It was thought that following the theory of Whang (1967) a slight 'pulling' or bulging might be seen in the wake toward the direction of the magnetic field lines when this direction did not coincide with the spacecraft velocity direction. Whang's theory dealt only with the ion distribution and did not determine the related electron distribution. Although 




Fig. 7(b).

our wake figures are not all symmetric they do not show a correlation with magnetic field direction. If it exists it is masked by other effects. This is particularly evident on comparing Fig. 7(a) with 7(b), and 8(a) with 8(b) which have the same magnetic field orientations. There is some bulging evident in Fig. 7(a) while Fig. 7(b) is much more symmetrical and similarly for $8(\mathrm{a})$ and $8(\mathrm{~b})$ where $8(\mathrm{~b})$ seems to be pulled somewhat in a direction away from $B$. 


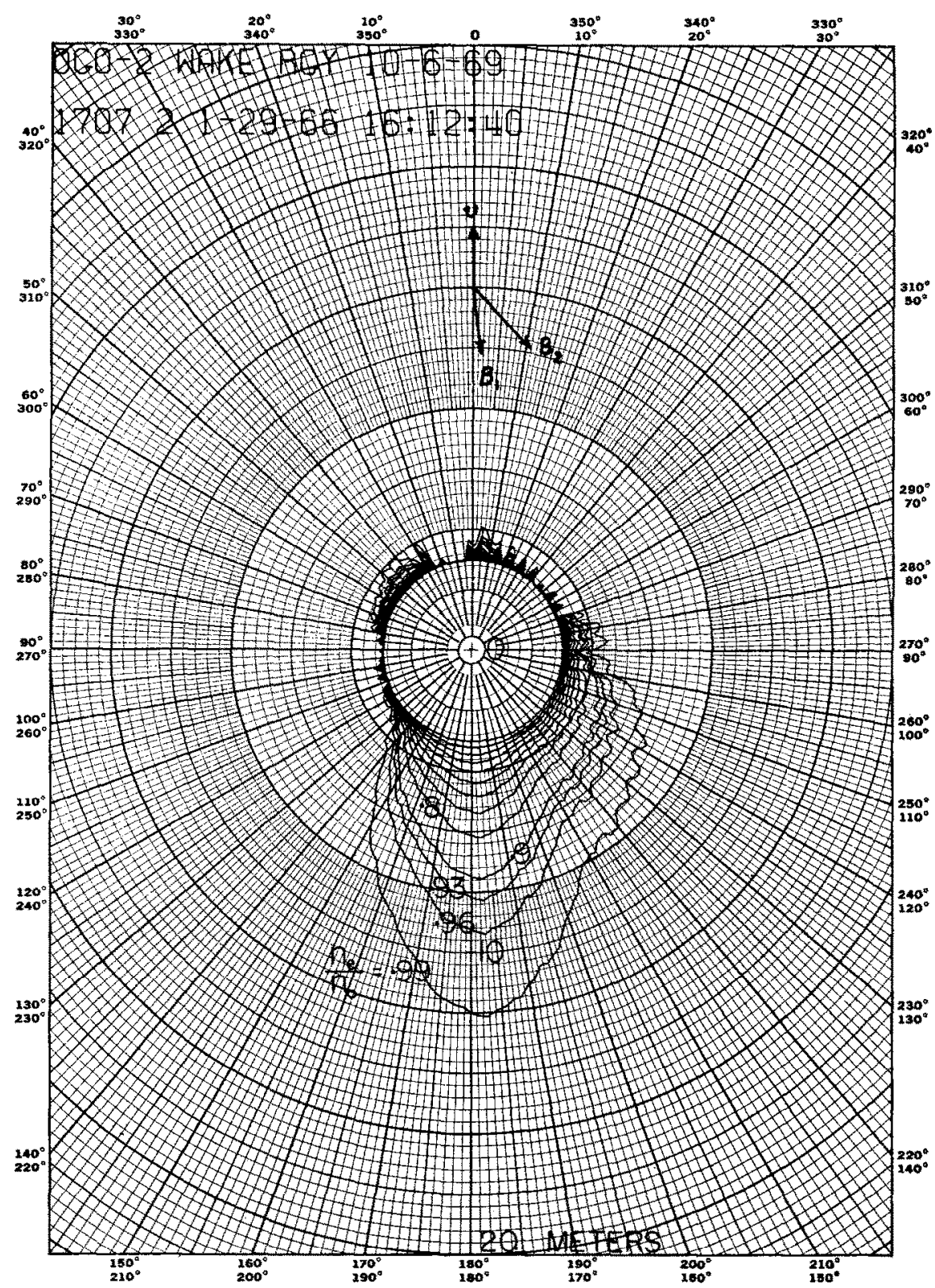

FIG. 8(a).

Fig. 8. Iso-relATIVE DENSTTY CONTOURS ILLUSTRATING TWO CASES WHEN $B_{1}$ AND $B_{2}$ ARE NOT SYMMETRIC ABOUT $V$.

The data presented here represents only a very small portion of all of the wake occurrences present in the raw data. Each perigec pass of the spacecraft could yield from one to five wake profiles and there are over thirteen perigee passes per $24 \mathrm{hr}$ day. Ionospheric disturbances during some perigee passes make part of the data unusable, but the clean passes contain more than enough data for additional analysis. Analysis of the type described plus additional tests of the correlation of wake parameters with magnetic field and altitude are being carried out. 


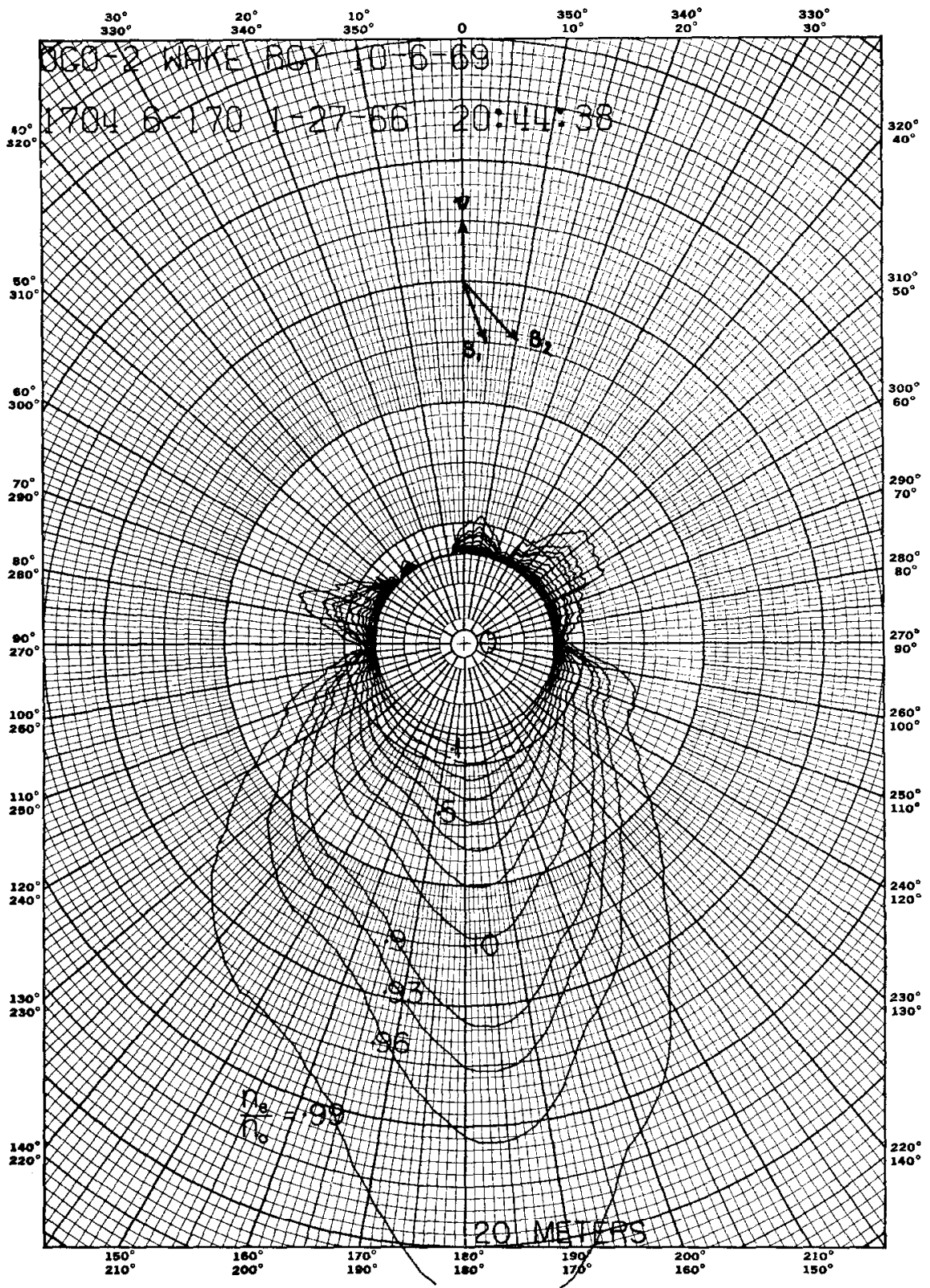

FIG. 8(b).

\section{CONCLUSIONS}

We have shown that the wake of a large spinning satellite can be effectively probed by an antenna since the effects of the wake on the impedance can be observed by an impedance bridge operating at a frequency slightly lower than the local plasma frequency. The results of this probing is an effective electron density distribution in the vicinity of the vehicle including the wake region. 
The axial length of the cavity for different angles of attack and the density distribution along the antenna has been found. If the satellite had been essentially symmetrical about the spin axis, the data would also have yielded the angular extent of the cavity. No correlation of wake shape with magnetic field direction was observed.

In addition to the electron density results, we have shown that, given suitable additional information, the spacecraft orientation can be determined from the impedance data.

The experiment was not designed for wake studies but gave useful results.

Our results indicate that an experiment could now be designed and built primarily to make accurate wake studies by antenna impedance measurements. In particular by using antennas of different lengths, one could verify whether the very near wake has an electron density dip as the self consistent theory states, rather than a monotonic increase with distance from the satellite.

Acknowledgements-The authors wish to thank Professor F. T. Haddock for his encouragement and support. They would also like to thank Professor V. C. Liu and Mr. W. H. Potter for useful discussions, and S. L. Breckenridge for her assistance in programming. This work was supported by the National Aeronautics and Space Administration under Grant No. NGR-23-005-068.

\section{REFERENCES}

AL'PERT, YA. L. (1965). Electromagnetic effects in the vicinity of an artificial satellite or space vehicle moving in the ionosphere or interplanetary space. Geomag. \& Aeronomy 5,1 .

Balmain, K. G. (1964). The impedance of a short dipole antenna in a magnetoplasma. IEEE Trans. AP-12, 605.

LIU, V. C. (1969). Ionospheric gas dynamics of satellite and diagnostic probes. Space Sci. Rev. 9, 423.

LuDwiG, G. H. and Scull, W. E. (1962). The orbiting geophysical observatory-new tool for space research. Astronautics 7, 24.

SAMR, U. and WILLMORE, A. P. (1965). The distribution of charged particles near a moving spacecraft. Planet. Space Sci. 13, 285.

SAWCHUCK, W. (1963). Wake of charged prolate spheroid at angle of attack in a rarefied plasma. Adv. Appl. Mech. Suppl. 2, 33. Rarefied Gas Dynamics 3rd Symp., Academic Press, New York.

Stone R. G., Fainberg, J. and Alexander, J. L. (1969). Measurements in the ionosphere of the aspect dependence of antenna reactance-I: Variations arising from the inhomogeneous plasma distribution about the vehicle. Planet. Space Sci. 17, 1437.

TRW Systems, INC. (1965). Operation summary Rep. No. OGO-II 14 October 1965-24 October 1965. Goddard Space Flight Center, OGO Exp. Bull. C-129, 8 December.

TRW Systems, Inc. (1966). OGO-II Operation Summary Report for February 1966. OGO Exp. Bull. C-207, 29 March.

WALSH, D. and HADDOCK, F. T. (1965). Antenna impedance in a plasma: problems relevant to radio astronomy measurements from space vehicles. Annls Astrophys. 28, 605.

WALSH, D. and WeIL H. (1968). Ionospheric focusing for an antenna in the presence of the geomagnetic field. Rudio Sci. 3 (New series), 936.

Whang, Y. C. (1968). Interaction of the magnetized solar wind with the Moon. Physics Fluids 11, 969. 